\title{
Production of a novel silk-like protein from sea anemone and fabrication of wet-spun and electrospun marine-derived silk fibers
}

\author{
Yun Jung Yang ${ }^{1,5}$, Yoo Seong Choi ${ }^{2,5}$, Dooyup Jung ${ }^{1}$, Byung Rak Park ${ }^{3}$, Woon Bong Hwang ${ }^{3}$, Hyun Woo Kim ${ }^{4}$ \\ and Hyung Joon Cha ${ }^{1}$
}

Silk-based biomaterials, which for centuries were primarily used as the raw materials for textile fabrics, are now being used in the medical, food and industrial fields because of their extraordinary properties. Their advantageous characteristics include high strength, extensibility, biocompatibility, biodegradability and minimal production of inflammatory reactions. To date, only silkworm silk has been applied in mass-produced technological applications, but spider silk proteins have been extensively studied because of their superior mechanical stability. Here we report the discovery of a novel silk-like protein (named aneroin) from the sea anemone Nematostella vectensis and the successful fabrication of wet-spun and electrospun silk fibers from purified recombinant aneroins. Aneroin fibers have promising mechanical properties, similar to those of recombinant spider silks with molecular weights of $\sim 100 \mathrm{kDa}$ and those of natural mammalian tendon collagen. The results of this study demonstrate that aneroin, a new repertoire of silk-like protein found in sea anemones, has potential for use as a novel fibrous biomaterial. Its use would expand the applications of silk in the development of multifunctional and bio-inspired materials. NPG Asia Materials (2013) 5, e50; doi:10.1038/am.2013.19; published online 7 June 2013

Keywords: electrospun nanofiber; marine silk fibers; recombinant silk proteins; sea anemone; wet-spun fiber

\section{INTRODUCTION}

Oceans cover approximately $70 \%$ of the Earth's surface, and $>95 \%$ of living organisms are found in ocean waters. ${ }^{1}$ However, $<1 \%$ of marine species have been cultured and examined for applications in medicine, industry and agriculture, ${ }^{2}$ which indicates that there may be an abundance of undiscovered but useful substances present in diverse marine organisms. One such useful biomaterial, silk, is used by a broad range of invertebrates as a building material for structures that capture prey or serve as protective cocoons; these structures are primarily composed of fibrous proteins. Recently, fibrous silk proteins have attracted attention as a biomaterial because of their superior biocompatibility and biofunctionality compared with synthetic polymers such as poly(ethylene oxide) and poly(E-caprolactone). ${ }^{3,4}$ Silkworm silk proteins, which have traditionally been used as raw materials for textile fabrics and biomedical sutures and are the most extensively characterized of all silk proteins, have been used as biomaterials and matrix scaffolds in the field of tissue engineering. ${ }^{5,6}$ Spider silk proteins have been the focus of the development of new biomimetic materials because of their characteristic properties of strength and extensibility and their ability to outperform most other natural and man-made fibers. Dragline silks, the primary structural web silks, exhibit exceptional toughness, similar to that of commercial polyaramid (aromatic nylon) filaments, and flagelliform silks are used for prey capture. ${ }^{7,8}$ However, there are currently no practical sources of silks other than silkworm silks. Therefore, the biotechnological production of recombinant silk proteins has been considered a promising alternative method for the mass production of silk materials. ${ }^{7,9}$ In addition, new biologically derived materials from marine organisms are expected to expand opportunities for a variety of medical and non-medical needs. ${ }^{10}$

Here we report the discovery of a novel silk-like protein in Nematostella vectensis (Figure 1a), a type of starlet sea anemone with large populations along the coasts of England and the United States. This sea anemone is widely used as a model organism of the phylum Cnidaria, which uses nematocysts for prey capture and defense. ${ }^{11}$ Nematocysts, the miniature weapons of cnidarians, are exocytotic organelles that are composed of a capsule and a coiled thread in the capsule that is used to capture prey in aquatic — primarily marineenvironments. Nematocysts are able to withstand extreme mechanical stress through a combination of high resistance and flexibility. ${ }^{12,13}$

${ }^{1}$ Department of Chemical Engineering, Pohang University of Science and Technology, Pohang, Korea; ${ }^{2}$ Department of Chemical Engineering, Chungnam National University, Daejeon, Korea; ${ }^{3}$ Department of Mechanical Engineering, Pohang University of Science and Technology, Pohang, Korea and ${ }^{4}$ Department of Marine Biology, Pukyong National University, Busan, Korea

Correspondence: Professor HJ Cha, Department of Chemical Engineering, Pohang University of Science and Technology, Pohang 790-784, Korea.

E-mail: hjcha@postech.ac.kr

5These authors contributed equally to this work.

Received 16 October 2012; revised 10 March 2013; accepted 11 March 2013 
We found a novel hypothetical protein (NCBI reference sequence, XP_001621085.1) in the full genome sequence of $N$. vectensis that is composed of 30 sets of highly repetitive decamers primarily composed of GPGNTGYPGQ, DPGNTGYPGQ and GPSNTGYPWQ sequences (Figure 1b). ${ }^{14}$ We expect that the protein can be used as a novel material for the fabrication of silk fibers with good mechanical properties.

\section{MATERIALS AND METHODS}

Sequence similarity search for the aneroin open-reading frame Regions of sequence similarity to the aneroin open-reading frame were investigated with a NCBI BLAST search of the protein in EMBL-EBI (http:// www.ebi.ac.uk/Tools/sss/ncbiblast/). The UniProt Knowledgebase databank was selected as the protein database. The open-reading frame sequence was uploaded as an input sequence, and the blastp program was used for the
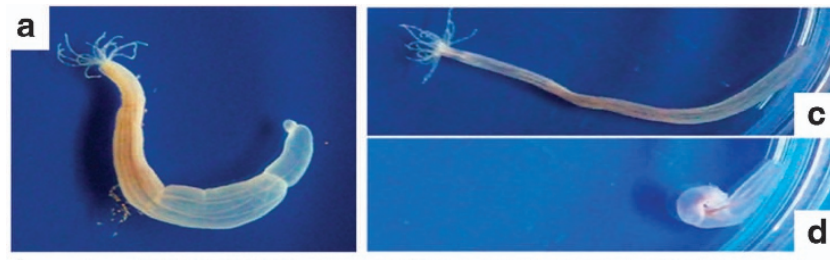

b 1 GPGNTGYPGQ GPGNTGHPGQ GPGNTGYPGQ DPGNTGYPGQ 41 DPGNTGYPGQ DPGNTGYPGQ GPGNTGCPGQ GPGNTGCPGQ 81 GPGNTGYPGQ GPGNTGYPGQ GPSNTGYPWQ GPGNT 116 GPGNTGYPGQ GPGNTGHPGQ GPGNTGYPGQ DPGNTGYPGQ 156 DPGNTGCPGQ GPGNTGCPGQ GSGNTGCPGQ GSGNTGCPGQ 196 GPGQ GPGNTGYPGQ GPGNTGHPGQ GPGNTGYPGQ 230 DPGNTGYPGQ DPGNTGCPGQ GPGNTGCPGQ GSGNTGCPGQ 270 GSGNTGCPGQ GPGQ GPGNTGYPGQ GPSNTGYPGQ 304 GPGNTGYPGQ GPGNTG
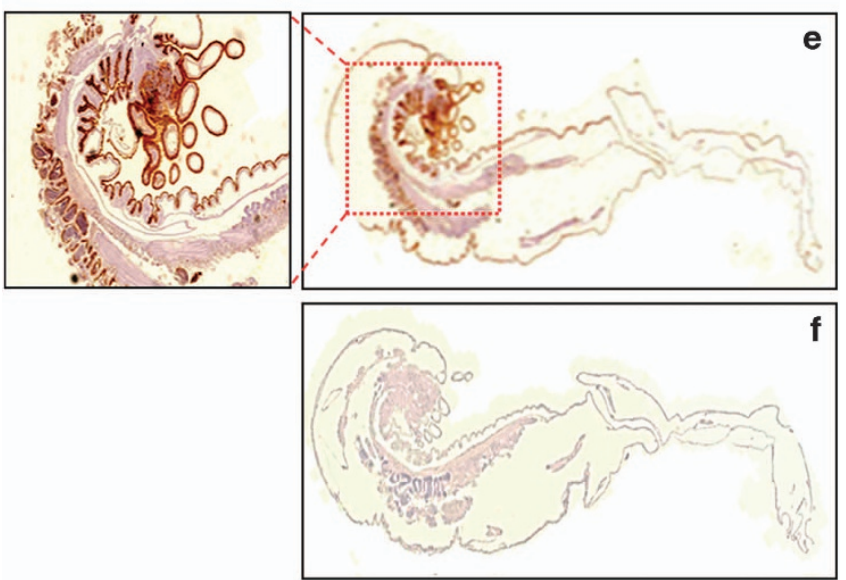

Figure 1 Aneroin, a novel silk-like protein from sea anemones. (a) $N$. vectensis. (b) Amino-acid sequence of the aneroin protein. $N$. vectensis images before (c) and after (d) stimulus. Immunohistochemical analyses of $N$. vectensis with aneroin-specific antibody (e) and purified normal rabbit IgG as a negative control (f). sequence similarity search. Table 1 summarizes the top-five sequence similarity search results for the hypothetical protein.

\section{Immunohistochemical analysis to locate the aneroin protein} in the sea anemone

To acquire an anti-aneroin antibody, a 15-mer partial peptide (PGQGPGNTGYPGQGP) of the aneroin protein was chemically synthesized (Young In Frontier, Seoul, Korea). Primary injection into a rabbit (New Zealand white) was conducted with $1 \mathrm{mg}$ of purified peptide, and subsequent boosting injections were performed twice with $500 \mu \mathrm{g}$ of peptide every 2 weeks. The rabbit anti-aneroin antibody was determined to have a titer of 100000 . $N$. vectensis was obtained from Dr Fabian Rentzsch (Sars International Centre for Marine Molecular Biology, Norway) and was incubated at room temperature in $12 \%$ artificial seawater. For immunohistochemical analysis, a sea anemone was fixed with Bouin's fixative solution overnight with mild agitation, dehydrated with ascending concentrations of ethanol solutions and cleared with toluene. The sea anemone was then cut into $4-\mu \mathrm{m}$ microtome-cut paraffin-embedded sections that were mounted on a positively charged coated slide (Surgipath, Richmond, IL, USA), deparaffinized in xylene and rehydrated with descending concentrations of ethanol solutions. This procedure was followed by heat-induced antigen retrieval, quenching of endogenous peroxidase activity with $0.3 \% \mathrm{H}_{2} \mathrm{O}_{2}$ for $30 \mathrm{~min}$ and blocking of non-specific binding with $4 \%$ bovine serum albumin. Sections were incubated with primary rabbit anti-aneroin antibody (1:200 000 dilution) at room temperature for $1 \mathrm{~h}$. After being washed, the sections were incubated with a secondary goat anti-rabbit IgG-conjugated with horseradish peroxidase (Dako, Carpinteria, CA, USA; 1:100 dilution) at room temperature for $1 \mathrm{~h}$. Irrelevant purified normal rabbit IgG (Invitrogen, Carlsbad, CA, USA), which should not have been present in the test sections, was used as a control. After 3,3'-diaminobenzidine (Dako) development, the sections were counterstained using hematoxylin (Sigma-Aldrich, St. Louis, MO, USA).

\section{Redesign of the aneroin sequence and vector construction}

To redesign the aneroin gene sequence, Escherichia coli codon preference was assumed to not converge dominantly on its major codons, especially those of glycine and proline, which are abundant in aneroin. Therefore, we adjusted the codons of the aneroin sequence by altering some major codons to other codons to avoid repetition. The aneroin gene, with an $\mathrm{N}$-terminal tryptophan operon leading (TrpL; MKAIFVLK) motif and an enterokinase cleavage site (DDDDK), was chemically synthesized (Integrated DNA Technologies, Commercial Park Coralville, IA, USA). The target sequence was introduced into a pET23b + vector (Novagen, Darmstadt, Germany), which contains a strong T7 promoter and hexahistidine sequence, that was predigested with NdeI and XhoI. Finally, the vector, pET-aneroin-30K, was constructed for the expression of recombinant aneroin-30K. To repeat the aneroin gene to double the protein's molecular weight, we used a SalI site that could be ligated to the fragment generated by XhoI digestion. Therefore, a PCR-amplified aneroin fragment with SalI and XhoI sites at each end was introduced into the XhoI-digested pET-aneroin-30K, providing the pET-aneroin-60K. All vector constructions were confirmed by direct sequencing.

\section{Expression and purification of recombinant aneroin proteins}

E. coli BL21(DE3) (Novagen) was used as a host for the expression of recombinant aneroin. Cells were cultured in Luria broth $(0.5 \%$ yeast extract,

Table 1 Top-five sequence similarity search results for the hypothetical protein

\begin{tabular}{|c|c|c|c|c|c|}
\hline Source & Proposed function & Identities & Positives & $D B: I D$ & E-value \\
\hline Pediculus humanus & Collagen alpha-1, IV, chain, putative & 47.0 & 55.0 & TR:EOVEK7_PEDHC & $2.0 \mathrm{E}-66$ \\
\hline Nephila inaurata madagascariensis & Flageliform silk protein (fragment) & 44.0 & 48.0 & TR:Q9NHW1_9ARAC & 3.0E-64 \\
\hline Nephila clavipes & Flageliform silk protein (fragment) & 44.0 & 45.0 & TR:Q9NHW4_NEPCL & $6.0 \mathrm{E}-63$ \\
\hline
\end{tabular}

Abbreviation: DB:ID, database identification on EMBL-EBI web services. 
$1 \%$ tryptophan and $1 \% \mathrm{NaCl}$ ) containing ampicillin (Sigma-Aldrich) at $37^{\circ} \mathrm{C}$, and protein expression was induced by application of $1 \mathrm{~mm}$ isopropyl- $\beta$-Dthiogalactopyranoside (Sigma-Aldrich) for $12 \mathrm{~h}$. Harvested cells were lysed using a sonic dismembrator (Thermo Fisher Scientific, Waltham, MA, USA) for $10 \mathrm{~min}$ at $50 \%$ power $(5 \mathrm{~s}$ pulse on and $5 \mathrm{~s}$ pulse off) in lysis buffer $(50 \mathrm{~mm}$ $\mathrm{NaH}_{2} \mathrm{PO}_{4}$ and $300 \mathrm{~mm} \mathrm{NaCl} ; \mathrm{pH} 8.0$ ), and the supernatant was collected after centrifugation at 4000 r.p.m. for $10 \mathrm{~min}$. The recombinant aneroin proteins were purified using two methods. Affinity-based purification was performed using Ni-NTA affinity chromatography (Qiagen, Valencia, CA, USA) under native conditions. The proteins were eluted using $750 \mathrm{~mm}$ imidazole solution ( $\mathrm{pH}$ 8.0), dialyzed against distilled water overnight and freeze-dried. For heatand acid-based purification, the soluble fraction was incubated at $42{ }^{\circ} \mathrm{C}$, and the resultant aggregates were collected by centrifugation at 9000 r.p.m. for $10 \mathrm{~min}$. The aneroin proteins were then extracted from the collected aggregates using 99\% formic acid (Samchun Chemical, Seoul, Korea), dialyzed against distilled water overnight and freeze-dried.

\section{Western blot analysis}

The sample was resolved by $12 \%(\mathrm{wt} / \mathrm{vol}$ ) SDS-PAGE. After the proteins were transferred onto a nitrocellulose membrane (Whatman, Maidstone, Kent, UK), the membrane was blocked with $5 \%(\mathrm{wt} / \mathrm{vol})$ non-fat dry milk for $1 \mathrm{~h}$. The proteins of interest were detected with a primary monoclonal anti-hexahistidine antibody (Santa Cruz Biotechnology, Santa Cruz, CA, USA; 1:1000 dilution) and a secondary anti-mouse IgG conjugated with alkaline phosphatase (Sigma-Aldrich; 1:1000 dilution). A color-development solution (Roche, Welwyn Garden City, UK) was added to detect the proteins of interest, and the reaction was quenched with distilled water.

\section{Matrix-assisted laser desorption/ionization time-of-flight mass spectrometry and amino-acid composition analyses}

Matrix-assisted laser desorption/ionization time-of-flight mass spectrometry (MALDI-TOF MS) was conducted using an Autoflex II MALDI-TOF system (Bruker Daltonics, Billerica, MA, USA) in the positive ion linear mode. Sinapinic acid in 50\% acetonitrile and $0.1 \%$ trifluoroacetic acid was used as the matrix solution. The samples were diluted 1:8 with the matrix solution, and the mixture was spotted onto the MALDI sample target plates and evaporated using a vacuum pump. Spectra were obtained in the mass range between 15000 and $130000 \mathrm{Da}$ with approximately 1000 laser shots, and calibration was performed using External protein standard II (Bruker Daltonics). The amino-acid analysis was performed using a Waters HPLC Pico-tag amino-acid analysis system (Waters Corporation, Milford, MA, USA). The sample was hydrolyzed with $6 \mathrm{~N} \mathrm{HCl}$ at $150{ }^{\circ} \mathrm{C}$ and derivatized with phenylisothiocyanate. Five microliters of the sample was injected at a flow rate of $1 \mathrm{ml} \mathrm{min}^{-1}$. Amino-acid standard H (Thermo Scientific Pierce, Waltham, MA, USA) was used, and the protein was detected under 254-nm absorbance.

\section{Determination of total carbohydrate and lipid amounts in purified aneroin samples}

To determine the total quantity of carbohydrates in the purified samples, a phenol-sulfuric acid assay was performed. ${ }^{15}$ Ten milligrams of each protein sample was dissolved in $500 \mu \mathrm{l}$ of $4 \%$ (wt/vol) phenol and $2.5 \mathrm{ml}$ of $96 \%$ (vol/vol) sulfuric acid. The optical density was measured at $490 \mathrm{~nm}$. For the standard curve, $1 \mathrm{mg} \mathrm{ml}^{-1}$ of glucose was used in $5-50 \mu \mathrm{l}$ of water. The sulfo-phospho vanillin colorimetric method was used to analyze the total quantity of lipids in the purified samples. ${ }^{16}$ Ten milligrams of each protein sample and 10-100 $\mu \mathrm{g}$ of triolein (standard sample) were dissolved in chloroform. The chloroform was dried, and $100 \mu \mathrm{l}$ of distilled water was added. The samples were mixed with $2 \mathrm{ml}$ of $18 \mathrm{M}$ sulfuric acid, boiled for $10 \mathrm{~min}$ in a water bath and cooled for $5 \mathrm{~min}$. Five milliliters of the phosphoric acidvanillin reagent $\left(85 \%(\mathrm{vol} / \mathrm{vol})\right.$ phosphoric acid and $1.2 \mathrm{gl}^{-1}$ vanillin) was treated and warmed at $37^{\circ} \mathrm{C}$ for $15 \mathrm{~min}$ and then cooled for $10 \mathrm{~min}$. After the reaction, the optical density was measured at $530 \mathrm{~nm}$ with a reference tube filled with water.

\section{Spinning of aneroin fibers and analyses of the mechanical} properties of fibers

1,1,1,3,3,3-Hexafluoroisopropanol was used as a spinning solvent. To obtain the spin dope, $10 \%$ (wt/vol) heat- and acid-purified aneroins were dissolved in 1,1,1,3,3,3-hexafluoroisopropanol. The dope solution was stirred and injected at a rate of $10 \mathrm{ml} \mathrm{h}^{-1}$ using a syringe pump (KD Scientific, Holliston, MA, USA) to coagulate in a bath containing $1: 1(\mathrm{vol} / \mathrm{vol})$ of isopropanol and methanol. The spun fiber was hand-drawn. The fiber samples were cut into $20-\mathrm{mm}$ sections to set a gauge length of $10 \mathrm{~mm}$, and each end was fixed securely onto paper. The paper was cut right before the tensile test. The tensile test was performed using a nano universal testing machine (MTS Nano Instruments, Oak Ridge, TN, USA) with 0.00027 strain per second of crosshead speed. The stress-strain curves were determined for analysis of the fibers' mechanical properties. The diameters of the aneroin fibers were measured with a micrometer (Mitutoyo, Kanagawa, Japan) and confirmed by a scanning electron microscope (JSM-7401F; JEOL, Akishima Tokyo, Japan) at an accelerating voltage of $5 \mathrm{kV}$.

To perform electrospinning, the aneroin proteins were dissolved in HFIP at a $10 \%(\mathrm{wt} / \mathrm{vol})$ concentration. Under high voltage $(11-13 \mathrm{kV})$, the spin dope was spun with a 23-gauge syringe at $0.5 \mathrm{ml} \mathrm{h}^{-1}$. The spun nanofibers were collected on aluminum foil. The distance between the syringe needle and the foil was approximately $10 \mathrm{~cm}$. The fiber morphology was assessed by scanning electron microscope, and the fiber diameters were measured using Image J software (National Institutes of Health, Bethesda, MD, USA). The electrospun samples were cut into rectangular sheets $\left(0.5 \times 2 \mathrm{~cm}^{2}\right)$. Their thickness and mechanical properties were measured in the same manner as for the wet-spun fibers. To investigate the enhancement of mechanical strength, some electrospun sheets were immersed in $100 \%$ (vol/vol) methanol for $30 \mathrm{~min}$ and dried for a day.

\section{RESULTS AND DISCUSSION}

\section{Sequence and immunohistochemical analyses}

More than 200 proteins comprise nematocyst capsules, primarily those of the mini-collagen protein family. ${ }^{12}$ The mini-collagens currently known are organized into three structural groups: those containing an $\mathrm{N}$-terminal cysteine-rich domain, those with glycineXaa-Xaa (GXX) repeats and those with a C-terminal cysteine-rich domain provided by extensive domain shuffling. ${ }^{12,13,17}$ However, the sequence pattern of our hypothetical protein is highly divergent from the sequences of previously reported capsule proteins in nematocysts, such as mini-collagens, Nowa proteins and spinalin. The sequence we discovered shares sequence identities of $48-55 \%$ with Bombyx mori fibrous proteins (GXX repeats), 47-48\% with collagens and 40-44\% with flagelliform spider silk proteins (GPGXX repeats) (Table 1). The body of an adult sea anemone is highly elastic and varies in length from $0.5 \mathrm{~cm}$ to $>10 \mathrm{~cm}$ (Figures $1 \mathrm{c}$ and $\mathrm{d}$ ). ${ }^{18}$ When a specific antibody was prepared using the 15-mer partial amino-acid sequence (PGQGPGNTGYPGQGP) of the hypothetical protein and this antibody was used for the immunohistochemical analysis of the protein's localization in the body of the sea anemone, positive signals were primarily distributed in the tentacles and were also detected to some degree on the surface of the body (Figures 1e-f). Based on the significant levels of glycine and proline in the nematocysts, the GXX repeat sequences of collagens, and the GPGXX repeats in spider silk proteins, ${ }^{13,19}$ we surmise that our hypothetical protein is also responsible for the formation of nematocysts and might be involved in the capture of prey.

\section{Biotechnological production of the silk-like protein from sea anemone}

The repetitive DNA sequence of the hypothetical protein (named aneroin) was redesigned for its successful biotechnological production with the aim of ensuring both genetic stability and efficient expression in E. coli. In addition, the TrpL sequence was fused at the $N$ terminus 
of aneroin to increase its expression. TrpL is a regulatory region, and its transcripts produce an alternate secondary structure that controls termination or attenuation at the transcription level. ${ }^{20}$ Although recombinant aneroin with a molecular weight of $\sim 32 \mathrm{kDa}$ (designated aneroin-30K) was successfully expressed in a primarily soluble form in E. coli, we found that it did not stain well in SDSPAGE, presumably because of its biased amino-acid composition and acidic nature. ${ }^{21}$ We confirmed the aneroin's hexahistidine-fused expression using western blot analysis with an anti-hexahistidine antibody (data not shown). After affinity-based chromatographic purification, the purity of the purified aneroin was indirectly analyzed by MALDI-TOF MS. Although some small peaks were observed, the peak $(\sim 32 \mathrm{kDa})$ corresponding to affinity-purified aneroin-30K was the primary peak (Figure 2a). Interestingly, the purified aneroin-30K proteins appeared in dimeric $(\sim 64 \mathrm{kDa})$ and tetrameric $(\sim 128 \mathrm{kDa})$ forms in our western blot (Figure $2 \mathrm{~b}$ ). To confirm purification, we performed amino-acid composition analysis and found that the experimental composition was quite similar to the calculated composition (Figure 2c). To investigate any enhancement of the proteins' mechanical properties, we also constructed a higher-molecular-weight $(\sim 62 \mathrm{kDa}$ ) aneroin protein (designated aneroin-60K; Figure $2 \mathrm{~d}$ ) by
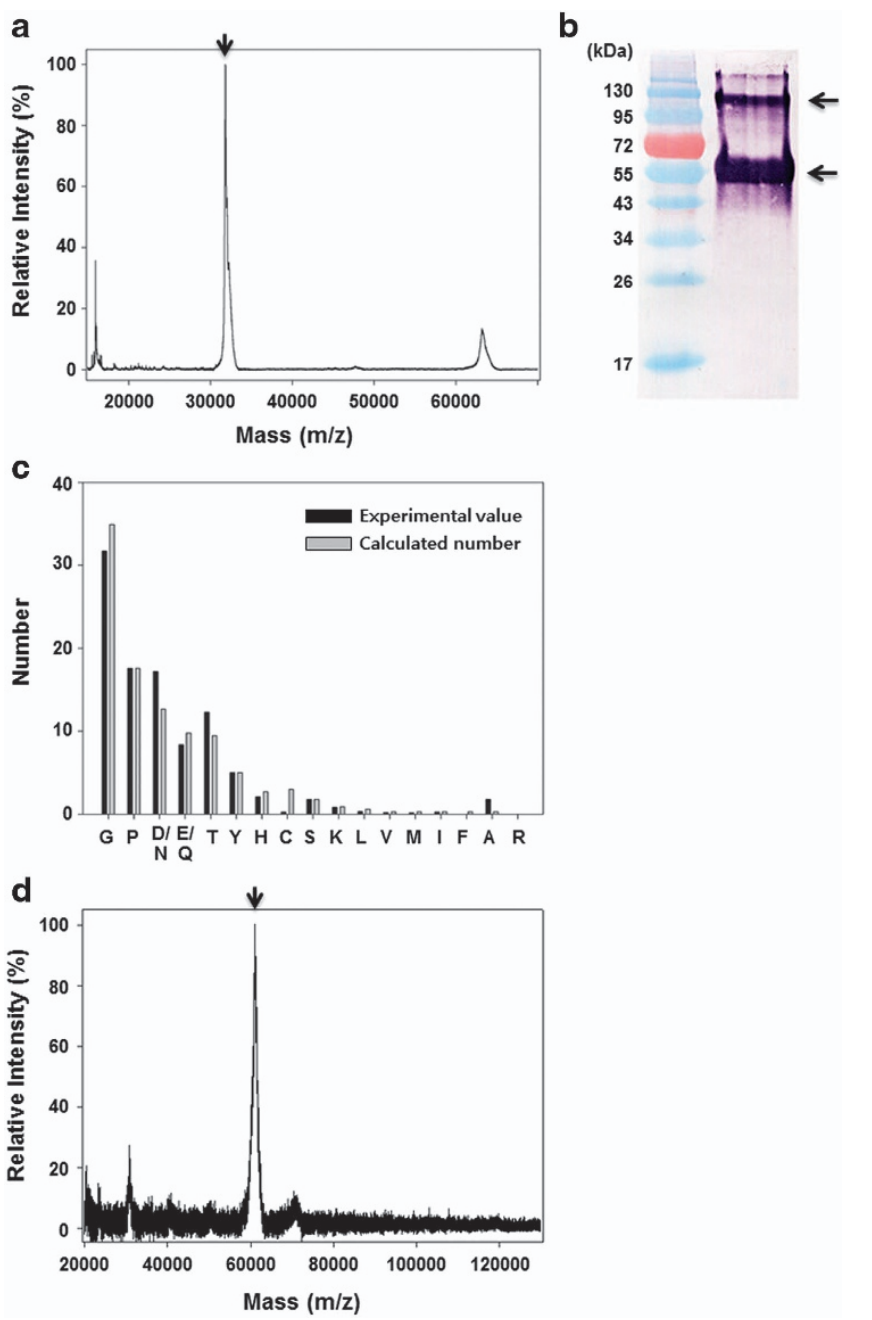

Figure 2 Expression and affinity-based purification of recombinant aneroin proteins. (a) MALDI-TOF MS, (b) western blot and (c) amino-acid composition analyses of aneroin-30K. (d) MALDI-TOF MS analysis of aneroin-60K.

repeating the aneroin gene twice, based on reports of the relationship between the molecular weights of recombinant spider silk proteins and the mechanical properties of the spun fibers. ${ }^{22,23}$

Materials with high-production capabilities and superior mechanical properties enable feasible and practical applications in various biomedical and industrial fields. Because affinity purification was not sufficient to obtain a high yield of recombinant aneroin proteins ( $\sim 67 \mu \mathrm{g} \mathrm{ml}^{-1}$ of aneroin-30K and $\sim 53 \mu \mathrm{g} \mathrm{ml}^{-1}$ of aneroin-60K), we devised an alternative method based on temperature-induced aggregation followed by acid extraction to improve purification yield and generate a simple purification procedure for practical applications. Elastin-like polypeptides, such as oligomeric repeats of the pentapeptide VPGXG, exhibit reversible and inverse phase transitions with varying temperatures. ${ }^{24}$ Recombinant spider silk proteins can be isolated by extraction with organic acids, such as formic acid and propionic acid. ${ }^{25}$ We found that the turbidity of the soluble fraction obtained by disruption of the aneroin-expressing cells increased substantially at temperatures $>42{ }^{\circ} \mathrm{C}$. Aneroin proteins were extracted from the temperature-induced aggregates using formic acid. This heat- and acid-based purification method provided samples with relatively high purities of the target aneroin proteins (see Figure $3 \mathrm{a}$ for aneroin-30K and Figure $3 \mathrm{~b}$ for aneroin-60K). Although the total quantities of carbohydrates and lipids in the heatand acid-based purified samples were 3-4 times higher than those in the affinity-purified samples, their percentages were minimal (Table 2). The heat- and acid-based purification method resulted in a purification yield approximately 4.5 times higher $\left(\sim 300 \mu \mathrm{g} \mathrm{ml}^{-1}\right.$ of aneroin-30K (Figure 3c) and $\sim 255 \mu \mathrm{g} \mathrm{ml}^{-1}$ of aneroin-60K (Figure 3d)) than the affinity purification method. Thus, our heat- and acid-based purification method provides a practical platform for the preparation of recombinant aneroin proteins in an effective and reproducible manner.

a

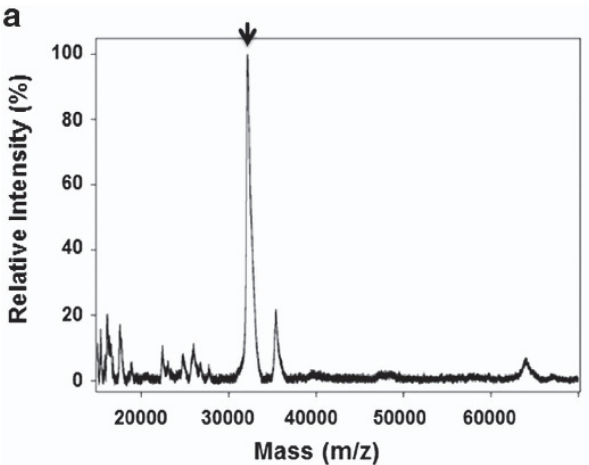

b

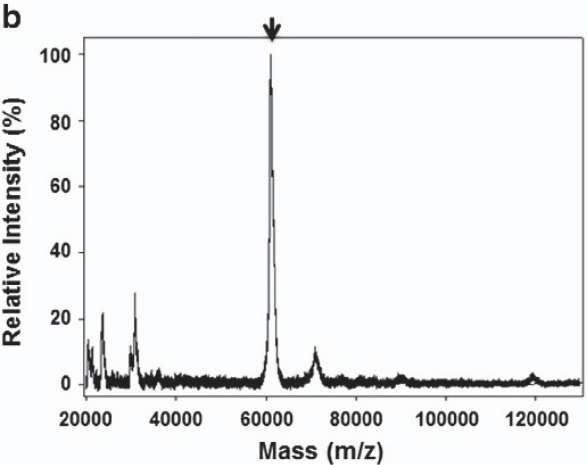

Figure 3 Heat- and acid-based purification of recombinant aneroin proteins. MALDI-TOF MS analyses of aneroin-30K (a) and aneroin-60K (b). Lyophilized powders of aneroin-30K (c) and aneroin-60K (d). 
The generation and processing of aneroin proteins into silk fibers The generation and processing of fibrous proteins into fibers are required for practical applications such as tissue engineering scaffolds, sutures and wound dressings. Wet-spinning is a characteristic and time-tested process for the preparation of silk fibers. Recently, electrospun nanofibers have been fabricated from synthetic and natural polymers, including fibrous proteins, for use in numerous applications. ${ }^{3}$ We fabricated silk-like fibers from recombinant aneroin proteins using both wet- and electrospinning methods. The morphological features and performance of silk fibers are dependent on the coagulation conditions, such as the temperature, $\mathrm{pH}$ and coagulant type and rate, that are used to form the fibers. ${ }^{6,26,27}$ Purified aneroin proteins were fully dissolved in HFIP and then extruded through a thin needle into a reservoir containing various organic solvents. The ability of the fibers to be spun was tested under various conditions by monitoring the breakage of the fibers and the microscale morphology of the resulting fibers (data not shown). As a result, the wet-spun aneroin silk fibers obtained from the heat- and acidpurified aneroin proteins were coagulated in a solution of 1:1 (vol/ vol) methanol/isopropanol (Figure $4 \mathrm{a}$ for aneroin-30K). Aneroin spun fibers of no-void circular cross-sections with diameters of $50-80 \mu \mathrm{m}$ were efficiently prepared at an extrusion rate of $10 \mathrm{ml} \mathrm{h}^{-1}$

Table 2 Comparison of the total amounts of lipids and carbohydrates in the affinity- and heat/acid-based purified samples

\begin{tabular}{lcc}
\hline Sample & $\begin{array}{c}\text { Lipids } \\
\left(\mu g \mathrm{mg}^{-1} \text { sample }\right)\end{array}$ & $\begin{array}{c}\text { Carbohydrates } \\
\left(\mu \mathrm{mg}^{-1} \text { sample }\right)\end{array}$ \\
\hline Affinity-based purification & 1.5 & 4.6 \\
Heat/acid-based purification & 4.1 & 21.1 \\
\hline
\end{tabular}

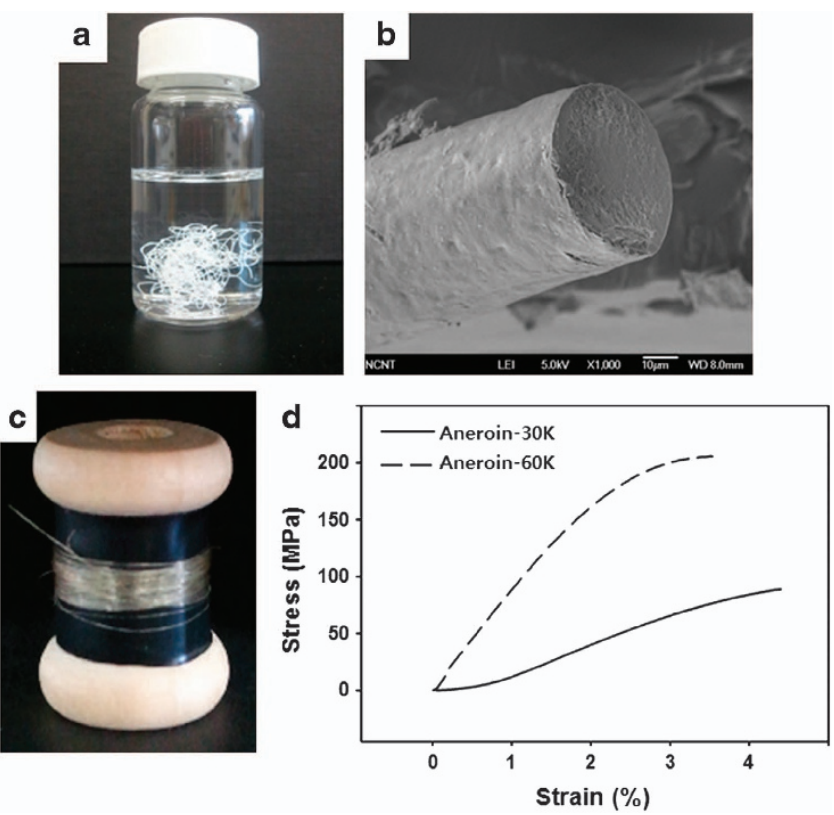

Figure 4 Aneroin fibers obtained through wet-spinning. (a) Coagulated wetspun aneroin-30K fibers in solution. (b) Scanning electron microscope analysis of wet-spun aneroin-30K fiber surfaces and a break point, used to examine the fiber's interior core (magnification $\times 1000$ ). (c) Aneroin-30K fiber skein. (d) Stress-strain curves of wet-spun aneroin-30K and aneroin$60 \mathrm{~K}$ fibers. (see Figure $4 \mathrm{~b}$ for an scanning electron microscope image of aneroin-30K and Figure $4 \mathrm{c}$ for a fiber skein of aneroin-30K). No notable morphological differences were observed between the aneroin-30K and aneroin-60K fibers. After hand-drawing fibers 2-3 times longer, the mechanical properties (strength, extensibility, stiffness and toughness) of the constructed aneroin silk fibers were characterized by stress-strain curves (Figure $4 \mathrm{~d}$ ) that were obtained using a nano universal testing machine (Table 3). The mechanical properties of the aneroin fibers were improved by increasing the molecular weights of the aneroin proteins. The aneroin fibers from aneroin-60K proteins displayed better mechanical properties ( $\sim 170 \mathrm{MPa}$ strength and $\sim 6.6 \mathrm{GPa}$ stiffness) than those of the aneroin-30K proteins ( $\sim 73 \mathrm{MPa}$ strength and $\sim 2.5 \mathrm{GPa}$ stiffness). Remarkably, the strength and stiffness of the aneroin fibers exceeded those of previously reported fibers produced from recombinant silk proteins with molecular weights of $\sim 60 \mathrm{kDa}$ in $E$. coli derived from major ampullate spider silk ( $\sim 2-6 \mathrm{MPa}$ strength and $\sim 0.0044 \mathrm{GPa}$ stiffness $)^{28}$ and from a mixture using flagelliform silk $(\sim 28-50 \mathrm{MPa}$ strength and $\sim 1.7 \mathrm{GPa}$ stiffness). ${ }^{29}$ The mechanical properties of aneroin fibers were similar to those of wet-spun fibers from recombinant spider silk proteins, with molecular weights of $\sim 100 \mathrm{kDa}$ having 32 repeats of the major ampullate spidroin I monomer, ${ }^{23}$ and from natural mammalian tendon collagen. ${ }^{30}$ We expect that the mechanical properties of aneroin fibers can be significantly enhanced by increasing the molecular weight of the recombinant aneroin proteins and/or by combining the aneroin protein with other nematocyst proteins, based on a putative model of nematocyst wall structure. ${ }^{13}$

We also constructed aneroin nanofibers from purified recombinant aneroin proteins using electrospinning. Ribbon-like nanofibers were spun from both aneroin proteins (see Figure $5 \mathrm{a}$ for aneroin-30K and Figure $5 \mathrm{~b}$ for aneroin-60K). The ribbon shape of these fibers might result from a thin skin formed by the rapid evaporation of the solvent and the collapse of the outer skin under atmospheric pressure. ${ }^{31}$ This ribbon shape has also been observed in elastin-mimetic peptide

Table 3 Mechanical properties of wet-spun aneroin fibers

\begin{tabular}{lcccc}
\hline Aneroin fiber & $\begin{array}{c}\text { Strength } \\
(\mathrm{MPa})\end{array}$ & $\begin{array}{c}\text { Extensibility } \\
(\%)\end{array}$ & $\begin{array}{c}\text { Stiffness } \\
(\mathrm{GPa})\end{array}$ & $\begin{array}{c}\text { Toughness } \\
\left(\mathrm{MJ} \mathrm{m}^{-3}\right)\end{array}$ \\
\hline Aneroin-30K & $73.04 \pm 14.92$ & $3.83 \pm 0.83$ & $2.46 \pm 0.57$ & $1.37 \pm 0.33$ \\
Aneroin-60K & $169.46 \pm 54.57$ & $3.35 \pm 0.60$ & $6.64 \pm 2.04$ & $3.66 \pm 1.70$ \\
\hline
\end{tabular}

A tensile test was performed using a nano universal testing machine, and strain-stress curves were collected for analysis of the mechanical properties of the fibers. Each measurement was repeated at least six times and averaged, reported here with one s.d.
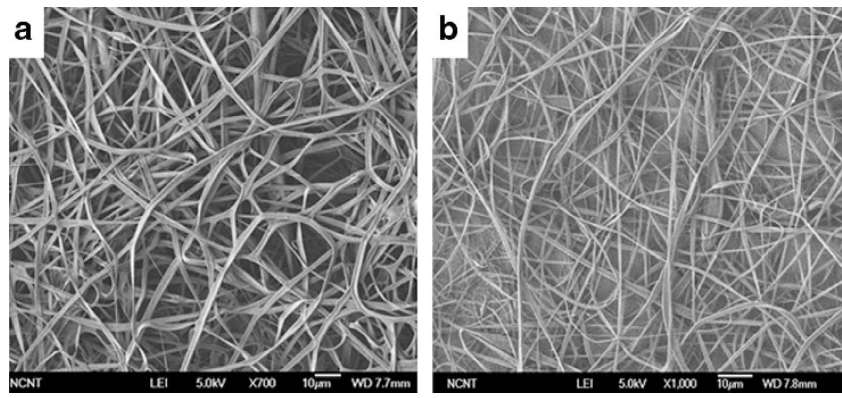

Figure 5 Aneroin nanofibers obtained through electrospinning. Scanning electron microscope images of electrospun (a) aneroin-30K and (b) aneroin$60 \mathrm{~K}$ nanofibers. 
Table 4 Mechanical properties of electrospun aneroin nanofiber sheets

\begin{tabular}{lcrcc}
\hline Aneroin sheet & $\begin{array}{c}\text { Strength } \\
(\mathrm{MPa})\end{array}$ & $\begin{array}{c}\text { Extensibility } \\
(\%)\end{array}$ & $\begin{array}{c}\text { Stiffness } \\
(\mathrm{MPa})\end{array}$ & $\begin{array}{c}\text { Toughness } \\
\left(\mathrm{MJ} \mathrm{m}^{-3}\right)\end{array}$ \\
\hline Aneroin-30K & $0.65 \pm 0.16$ & $21.14 \pm 5.56$ & $8.03 \pm 2.88$ & $0.36 \pm 0.31$ \\
Aneroin-60K & $3.93 \pm 0.44$ & $50.97 \pm 6.86$ & $52.07 \pm 9.12$ & $1.71 \pm 0.34$ \\
m-Aneroin-60Ka & $59.46 \pm 15.12$ & $5.12 \pm 0.80$ & $1,845 \pm 404$ & $1.54 \pm 0.48$ \\
\hline
\end{tabular}

Sample was cut into rectangle-shape sheet $\left(0.5 \times 2 \mathrm{~cm}^{2}\right)$. A tensile test was performed using a nano universal testing machine, and strain-stress curves were collected for analysis of the mechanical properties of the sheets. Each measurement was repeated at least five times and averaged, reported here with one s.d.

aMethanol-treated aneroin-60K nanofiber sheet.

polymers. ${ }^{32}$ In addition, the width of the nanofiber could be adjusted by changing the concentration of the spin dope. A $10 \%$ (wt/vol) spin dope resulted in electrospun nanofibers, with a dominant width of approximately $2000 \mathrm{~nm}$ and a width of approximately $700 \mathrm{~nm}$ in $7.5 \%$ (wt/vol) solution. However, nanofibers were not successfully spun under a $5 \%(\mathrm{wt} / \mathrm{vol})$ spin dope. After the electrospun nanofiber samples were cut into rectangular sheets, their mechanical properties were measured (Table 4). The aneroin-60K nanofibers exhibited better mechanical properties ( $\sim 4 \mathrm{MPa}$ strength and $\sim 52 \mathrm{MPa}$ stiffness) than the aneroin-30K nanofibers $(\sim 0.7 \mathrm{MPa}$ strength and $\sim 8 \mathrm{MPa}$ stiffness). As in the case of the wet-spun fibers, increasing the molecular weight is a feasible strategy for ameliorating the mechanical features of electrospun nanofibrous materials. Because it has been reported that the mechanical properties of silkworm silk sheets can be improved using $100 \%$ methanol, ${ }^{33}$ we also investigated the effect of methanol on the mechanical properties of the aneroin silk nanofibers. We found that methanol-submerged aneroin-60K nanofibers ( $m$-aneroin-60K) exhibited significantly enhanced mechanical properties, especially strength $(\sim 60 \mathrm{MPa})$ and stiffness $(\sim 1.8 \mathrm{GPa})$ (Table 4$)$. These values are roughly similar to those for wet-spun aneroin-30K fibers but are much less than the values for wet-spun aneroin-60K fibers. Because electrospun nanofiber sheets include many inner pores, the measured thickness is much greater than the actual thickness, which might result in distinct differences in the mechanical properties of fibers obtained using the two spinning methods. ${ }^{34}$ Recently, the fabrication of electrospun fibers with various mechanical and biological properties in a targeted manner has been a subject of interest. We expect that aneroins can be used to develop a novel type of biopolymer-based nanofiber that could be used in various applications, including tissue engineering scaffolds, wound healing and drug release/transport biomaterials.

\section{CONCLUSIONS}

We have demonstrated for the first time the generation of silk fibers using a novel silk-like protein, aneroin, that originates from the sea anemone N. vectensis. The sequence of decapeptide repeats in aneroin is highly similar to the sequences of other fibrous proteins, including silkworm silks, spider silks and collagens. We found that the aneroin protein is primarily located in the nematocysts, indicating that it might be involved in the formation of nematocyst wall structures, in combination with other reported nematocyst proteins. We produced a feasible quantity of recombinant aneroin proteins in E. coli through the genetic redesign of the aneroin sequence, and we successfully fabricated the protein into silk fibers by both wet-spinning and electrospinning. The aneroin fibers exhibited mechanical properties similar to those of both recombinant spider silks (with molecular weights of $\sim 100 \mathrm{kDa}$ ) and natural mammalian tendon collagen. Thus, we expect that silk fibers from the sea anemone have great potential for use in the development of bio-inspired natural materials, and we believe that their mechanical properties and biocompatibility make them well suited for many practical applications.

\section{ACKNOWLEDGEMENTS}

This work was supported by the Marine Biotechnology Program, funded by the Ministry of Land, Transport and Maritime Affairs of Korea and the Rising Star Program funded by POSTECH. We thank Dr F Rentzsch (Sars Centre, Norway) for providing us with Nematostella vectensis, Drs H-S Lee (KORDI, Korea) and K-S Choi (JNU, Korea) for their useful advice on sea anemone, and Y-H Park (SNU, Korea) and K H Lee (SNU, Korea) for their useful advice on wet-spinning.

Author contributions: YJY, YSC and HJC designed the experiments. YJY, YSC and DJ carried out the experiments. YJY, YSC, BRP, WBH, HWK and HJC contributed to analysis of the data. YJY, YSC and HJC wrote the manuscript.

1 Angel, M. V. Biodiversity of the pelagic ocean. Conserv. Biol. 7, 760-772 (1993).

2 Committee on Marine Biotechnology Marine Biotechnology in the Twenty-first Century: Problems, Promise, and Products 1-100 (The National Academies Press, Washington, 2002).

3 Greiner, A. \& Wendorff, J. H. Electrospinning: a fascinating method for the preparation of ultrathin fibers. Angew. Chem. Int. Ed. 46, 5670-5703 (2007).

4 Kaplan, D. L., Adams, W. W., Farmer, B. \& Viney, C. in Silk Polymers: Materials Science and Biotechnology (eds Kaplan, D. L., Adams, W. W., Farmer, B. \& Viney, C.) 2-16 (American Chemical Society, Washington, 1994).

5 Altman, G. H., Diaz, F., Jakuba, C., Calabro, T., Horan, R. L., Chen, J., Lu, H., Richmond, J. \& Kaplan, D. L. Silk-based biomaterials. Biomaterials 24, 401-416 (2003).

6 Shao, Z. \& Vollrath, F. Surprising strength of silkworm silk. Nature 418, 741 (2002).

7 Heim, M., Keerl, D. \& Scheibel, T. Spider silk: from soluble protein to extraordinary fiber. Angew. Chem. Int. Ed. 48, 3584-3596 (2009).

8 Vollrath, F. \& Knight, D. P. Liquid crystalline spinning of spider silk. Nature 410 541-548 (2001)

9 Omenetto, F. G. \& Kaplan, D. L. New opportunities for an ancient material. Science 329, 528-531 (2010).

10 Ehrlich, H. Biological Materials of Marine Origin 3-21 (Springer, Berlin, 2010).

11 Darling, J. A., Reitzel, A. R., Burton, P. M., Ryan, J. F., Sullivan, J. C. \& Finnerty, J. R Rising starlet: the starlet sea anemone, Nematostella vectensis. Bioessays 27, 211-221 (2005).

12 Ozbek, S., Balasubramanian, P. G. \& Holstein, T. W. Cnidocyst structure and the biomechanics of discharge. Toxicon 54, 1038-1045 (2009).

13 David, C. N., Ozbek, S., Adamczyk, P., Meier, S., Pauly, B., Chapman, J., Hwang, J. S., Gojobori, T. \& Holstein, T. W. Evolution of complex structures: minicollagens shape the cnidarian nematocyst. Trends Genet. 24, 431-438 (2008).

14 Putnam, N. H., Srivastava, M., Hellsten, U., Dirks, B., Chapman, J., Salamov, A., Terry, A., Shapiro, H., Lindquist, E., Kapitonov, V. V., Jurka, J., Genikhovich, G. Grigoriev, I. V., Lucas, S. M., Steele, R. E., Finnerty, J. R., Technau, U., Martindale, M. Q. \& Rokhsar, D. S. Sea anemone genome reveals ancestral eumetazoan gene repertoire and genomic organization. Science 317, 86-94 (2007)

15 Fournier, E. in Current Protocols in Food Analytical Chemistry (ed. Wrolstad, W. E). Ch. E, E1.1.1-E1.1.8 (John Wiley and Sons, Hoboken, 2001).

16 Izard, J. \& Limberger, R. J. Rapid screening method for quantitation of bacterial cell lipids from whole cells. J. Microbiol. Methods 55, 411-418 (2003).

17 Holstein, T. W., Benoit, M., Herder, G. V., David, C. N., Wanner, G. \& Gaub, H. E. Fibrous mini-collagens in hydra nematocysts. Science 265, 402-404 (1994).

18 Trevino, M., Stefanik, D. J., Rodriguez, R., Harmon, S. \& Burton, P. M. Induction of canonical Wnt signaling by alsterpaullone is sufficient for oral tissue fate during regeneration and embryogenesis in Nematostella vectensis. Dev. Dyn. 240, 2673-2679 (2011)

19 Kluge, J. A., Rabotyagova, O., Leisk, G. G. \& Kaplan, D. L. Spider silks and their applications. Trends Biotechnol. 26, 244-251 (2008).

20 Bandmann, N. \& Nygren, P. A. Combinatorial expression vector engineering for tuning of recombinant protein production in Escherichia coli. Nucleic Acids Res 35, e32 (2007).

21 Goldberg, H. A. \& Warner, K. J. The staining of acidic proteins on polyacrylamide gels: enhanced sensitivity and stability of 'Stains-all' staining in combination with silver nitrate. Anal. Biochem. 251, 227-233 (1997).

22 Xia, X. X., Qian, Z. G., Ki, C. S., Park, Y. H., Kaplan, D. L. \& Lee, S. Y. Native-sized recombinant spider silk protein produced in metabolically engineered Escherichia coli results in a strong fiber. Proc. Natl Acad. Sci. USA. 107, 14059-14063 (2010).

23 Northolt, M. G., den Decker, P., Picken, S. J., Baltussen, J. J. M. \& Schlatmann, R. The tensile strength of polymer fibres. Adv. Polym. Sci. 178, 1-108 (2005).

24 Meyer, D. E. \& Chilkoti, A. Purification of recombinant proteins by fusion with thermally-responsive polypeptides. Nat. Biotechnol. 17, 1112-1115 (1999). 
25 Mello, C. M., Soares, J. W., Arcidiacono, S. \& Butler, M. M. Acid extraction and purification of recombinant spider silk proteins. Biomacromolecules 5, 1849-1852 (2004).

26 Teulé, F., Cooper, A. R., Furin, W. A., Bittencourt, D., Rech, E. L., Brooks, A. \& Lewis, R. V. A protocol for the production of recombinant spider silk-like proteins for artificial fiber spinning. Nat. Protoc. 4, 341-355 (2009).

27 Chen, X., Shao, Z. \& Vollrath, F. The spinning processes for spider silk. Soft Matter 2 448-451 (2006).

28 Brooks, A. E., Stricker, S. M., Joshi, S. B., Kamerzell, T. J., Middaugh, C. R. \& Lewis, R. V. Properties of synthetic spider silk fibers based on Argiope aurantia MaSp2. Biomacromolecules 9, 1506-1510 (2008).

29 Teulé, F., Furin, W. A., Cooper, A. R., Duncan, J. R. \& Lewis, R. V. Modifications of spider silk sequences in an attempt to control the mechanical properties of the synthetic fibers. J. Mater. Sci. 42, 8974-8985 (2007).

30 Pollock, C. M. \& Shadwick, R. E. Relationship between body mass and biomechanical properties of limb tendons in adult mammals. Am. J. Physiol. 266, R1016-R1021 (1994).
31 Koombhongse, S., Liu, W. \& Reneker, D. H. Flat polymer ribbons and other shapes by electrospinning. J. Polym. Sci. Pol. Phys. 39, 2598-2606 (2001).

32 Huang L., McMillan, R. A A Apkarian, R. P Pourdeyhimi, B., Conticello, V. P. \& Chaikof, E. L. Generation of synthetic elastin-mimetic small diameter fibers and fiber networks. Macromolecules 33, 2989-2997 (2000).

33 Um, I. C., Kweon, H., Lee, K. G., Ihm, D. W., Lee, J.-H. \& Park, Y. H. Wet spinning of silk polymer: I. Effect of coagulation conditions on the morphological feature of filament. Int. J. Biol. Macromol. 34, 89-105 (2004).

34 Wang, M., Jin, H. J., Kaplan, D. L. \& Rutledge, G. C. Mechanical properties of electrospun silk fibers. Macromolecules 37, 6856-6864 (2004).

(c) (i) $($ ) $\Theta$ This work is licensed under a Creative Commons Attribution-NonCommercial-NoDerivs 3.0 Unported License. To view a copy of this license, visit http://creativecommons. org/licenses/by-nc-nd/3.0/ 